\section{Idle No More for First Nations Rights}

A new protest movement for First Nations rights, started in the wake of Parliament of Canada's adoption last fall of Omnibus Bill C-45, is gaining momentum across the country. Inspired in part by the hunger strike of Attawapiskat First Nation Chief Theresa Spence, the protest takes aim at the disregard for Aboriginal rights in that Bill, particularly the deregulation of waterways in the country, many of which pass through Aboriginal lands. This deregulation may facilitate industrial development such as oil pipelines around fragile ecosystems in remote areas and on First Nations' land, threatening the environment as well as the health and well-being of local populations.

We must use this opportunity to take a much deeper and broader look at the state of Aboriginal health and well-being in Canada. Living standards, health status and quality of life are still unacceptably poor in many remote First Nations communities, and prospects for social and economic security for Aboriginal People living in many parts of the country are bleak.

Public health can be idle no more. For a country looked to worldwide for its contributions in defining the social determinants of health, Canada has failed miserably in addressing its most important social determinant, and this open wound to our country should be a major health care and public health priority. We need a "Marshall Plan" for First Nations in Canada. Not a Marshall Plan that sends cash to communities, but one that develops jointly with First Nations a common vision for the social, economic and health development of First Nations over the next several decades. A Marshall Plan that harnesses the intellectual, industrial, communication, infrastructure, financial and social capital of Canadian society, and brings in the public and private sectors in a concerted effort to build a better future for our First Nations.

Based on an overarching national public policy framework to address the educational, housing, social, health, economic and other needs of First Nations, this plan will require substantial resources as well as public health input for research, programs, evaluation, surveillance, organization of services, etc. Research on the impact of policies and programs based on participatory research principles will be critical for the success of these endeavours.

Calling for a Marshall Plan is easy to do. It is an oversimplification to imply that an all-encompassing initiative will solve First Nations problems. We all know that we are dealing with complex issues and that many years and much more than money will ultimately be required. Changes in mentality, capacity building and respect for the autonomy of First Nations will be critical to the success of any attempt to address their determinants of health. However, we have a moral imperative to act, we have the means to achieve our goals and we have a debt to our First Nations. Who will lead?

Gilles Paradis

Scientific Editor

\section{Jamais plus l'inaction à l'égard des droits des Premières Nations}

Un nouveau mouvement de protestation en faveur des droits des Premières Nations, né dans la foulée de l'adoption par le Parlement du Canada, l'automne dernier, de la loi omnibus C-45, prend de l'ampleur dans tout le pays. Inspiré en partie par la grève de la faim de la chef de bande d'Attawapiskat, Theresa Spence, le mouvement prend pour cible le peu de cas fait des droits des Autochtones dans le projet de loi, en particulier la déréglementation des voies navigables du pays, dont beaucoup passent par des terres autochtones. Cette déréglementation pourrait faciliter le développement industriel, comme les oléoducs qui contournent des écosystèmes fragiles dans des régions isolées et sur les terres des Premières Nations, menaçant l'environnement, ainsi que la santé et le bien-être des populations locales.

Nous devons saisir cette occasion pour approfondir et élargir notre examen de l'état de santé et de bien-être des Autochtones au Canada. Le niveau de vie, l'état sanitaire et la qualité de vie sont encore inacceptables dans de nombreuses communautés reculées des Premières nations, et les perspectives de sécurité sociale et économique sont sombres pour les Autochtones dans bien des coins du pays.

La santé publique ne peut plus se passer d'agir. Pour un pays admiré de par le monde pour son rôle dans la définition des déterminants sociaux de la santé, le Canada échoue lamentablement à aborder son déterminant social le plus important; cette plaie vive pour notre pays devrait être une priorité absolue dans les domaines des soins de santé et de la santé publique. Nous avons besoin d'un " plan Marshall » pour les Premières Nations au Canada. Pas un plan Marshall qui envoie de l'argent aux communautés, mais qui façonne avec les Premières Nations une vision commune de leur développement social, économique et sanitaire pour les décennies à venir. Un plan Marshall qui mobilise le capital intellectuel, industriel, communicationnel, infrastructurel, financier et social de la société canadienne, et qui amène les secteurs public et privé à agir de concert pour que nos Premières Nations aient un avenir meilleur.

Fondé sur un cadre national de politiques publiques chapeautant les besoins des Premières Nations en matière d'éducation et de logement, en matière sociale, sanitaire, économique et autres, un tel plan nécessitera des ressources considérables, ainsi que l'apport de la santé publique pour la recherche, les programmes, l'évaluation, la surveillance, l'organisation des services, etc. La recherche sur l'impact des politiques et des programmes, basée sur les principes de la recherche participative, sera essentielle à la réussite de cette entreprise.

Il est facile de réclamer un plan Marshall. C'est simplifier à outrance que de laisser entendre qu'une initiative globale résoudra les problèmes des Premières Nations. Nous savons tous que nous sommes aux prises avec des enjeux complexes, et qu'il faudra des années, et beaucoup plus que de l'argent, pour espérer les résoudre. Un changement des mentalités, le renforcement des capacités et le respect de l'autonomie des Premières Nations seront indispensables à la réussite de toute tentative pour aborder les déterminants de leur santé. Nous avons cependant l'impératif moral d'agir, nous avons les moyens d'atteindre nos objectifs et nous avons une dette envers nos Premières Nations. Qui donnera l'exemple?

Le rédacteur scientifique, Gilles Paradis 